\title{
Fabric Defect Identification Based on 2D Empirical Mode Decomposition
}

\author{
Shuaijun Wang, Ye Yuan* and Hongli Dun \\ School of Information Engineering, Beijing Institute of Fashion Technology, Beijing 100029, China \\ ${ }^{*}$ Corresponding author
}

\begin{abstract}
Fabric defect identification has constituted a key part in the online quality control regarding the textile manufacturing process. Focusing on the limitations of conventional fabric defect identification methods in representing local geometry of fabric defects, a fully adaptive image processing technique called twodimensional empirical mode decomposition is introduced in this paper to extract the identification feature of fabric defect and segment the fabric defect region. The firstly decomposed intrinsic mode component is capable of maintaining fine-scale patterns of the fabric defect, while the secondly decomposed intrinsic mode component possesses the main energy of medium-scale patterns. As a result, both energy and local scale parameters along warp or weft direction are derived from either first intrinsic mode component or second intrinsic mode component to locate the defect pixels in the fabric. Simulation results have shown that the proposed method is characterized by high accuracy with fast computational speed.
\end{abstract}

Keywords-2D empirical mode decomposition; intrinsic mode component; fabric defect

\section{INTRODUCTION}

Normal fabric is composed of warp and weft on a loom ups and downs with each other according to certain rules interwoven warp and weft threads cross each other or each other's ups and downs law constitutes normal tissue fabric surface, as shown in Figure I (a). Fabric defects in the weaving process warp and weft yarns in itself defective, oil on fabric rendered weaken its quality and affect the appearance of defects in materials or irregular, as shown in Figure I (a) - (d).

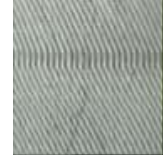

(a)

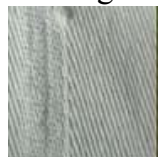

(b)

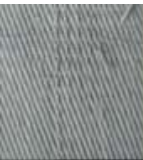

(c)

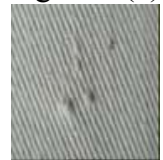

(d)
FIGURE I. FABRIC WEAVES AND FABRIC DEFECTS

The current approach[1-5] has limitations fabric defect in the following areas: (i) Assumed to be treated fabric image is smooth, with fabric image inherent characteristics contrary to the non-stationary .Such as statistical methods. (ii) Lack of space-frequency characteristics of local and multi-scale characteristics reflect the human visual system. Such as the method of Fourier transform and the model-based approach. (iii) Not completely determine the local scale adaptive features and data driving characteristics. Such as the method of wavelet, Gabor filter. Linear multi-scale analysis can overcome the limitations of these three aspects to a certain extent, the linear multi-scale analysis as the primary means for detecting fabric defects, which are able to use the advantages of the joint fabric image feature information on each scale unified analysis. However, fabric defect essentially nonstationary, normal weave fabric defects and have a completely different texture characteristics of different fabric textures joint feature analysis does not adapt to the requirements of non-stationary fabric defects on the specified scale. Nonlinear multi-scale analysis method recently proposed a twodimensional empirical mode decomposition [6-8] as the representative to the local spatial scale signal, rather than the default basis function based on signal decomposition, it is possible to characterize the fine signal inherent in space frequency structure important way, especially for non-linear, non-stationary signals, to overcome the limitations of existing methods to solve fabric defect deal with the problem.

\section{2D EMPIRICAL MODE DECOMPOSITION}

2D on EMD complex fabric image can be decomposed into different "layers of tissue," the upcoming multicomponent fabric structure is divided into several different scales of single-component structure, every single component of the organizational structure are in line with the intrinsic mode function 2D zero-mean AM-FM signal definition. Wherein the first-order intrinsic mode components with high frequency resolution, the image corresponding to the fabric of the most sophisticated organizational structure; second order modal components intrinsic resolution of the general level or lower, corresponding to the image coarser fabric organizational structure. Assumed to be decomposed fabric image to achieve the 2D empirical mode decomposition algorithm is as follows:

Step1. Initialization: the remaining components $\mathrm{R}_{0}(\mathrm{~m}, \mathrm{n})=\mathrm{P}(\mathrm{m}, \mathrm{n})$, intrinsic mode components of the orderj $=1$.

Step2.Executive the screening operation of $j$-order intrinsic mode components.

Step2.1 Order $I_{0}(m, n)=R_{j-1}(m, n), k=1$.

Step2.2 Search the local maxima and local minima ofl $_{\mathrm{k}-1}(\mathrm{~m}, \mathrm{n})$.

Step 2.3 Using radial basis function, or hierarchy of Bsplines [9-10], or finite element [11-12], respectively, of the local maxima and local minima are interpolated surface structure of the upper envelope and the lower envelope surface.

Step 2.4 According to the following formula to calculate the average Surface: 


$$
\mathrm{M}_{k-1}(m, n)=\frac{\mathrm{S}_{k-1, u}(m, n)+\mathrm{S}_{k-1, l}(m, n)}{2}
$$

Step 2.5 Subtracte $M_{k-1}(m, n)$ from $I_{k-1}(m, n)$, that is

$$
\mathrm{I}_{k}(m, n)=\mathrm{I}_{k-1}(m, n)-\mathrm{M}_{k-1}(m, n)
$$

Step 2.6 Order $\mathrm{k}=\mathrm{k}+1$, repeat Step 2.2 to Step 2.5 until the following criteria are met:

$$
e=\sum_{m, n}\left[\frac{\left|\mathrm{I}_{k}(m, n)-\mathrm{I}_{k-1}(m, n)\right|^{2}}{\mathrm{I}_{k}^{2}(m, n)}\right]<\varepsilon
$$

Among them , $\varepsilon$ is the pre-defined threshold.

Step 3. If the expression (3) is satisfied, then $I_{k}(m, n)$ is the j-order of intrinsic mode components of $P(m, n)$ and expressed as $C_{j}(m, n)=I_{k}(m, n)$.

Step 4. Subtract $C_{j}(m, n)$, the j-order of intrinsic mode components, from $\mathrm{R}_{\mathrm{k}-1}(\mathrm{~m}, \mathrm{n})$, update the remaining components. which is:

$$
\mathrm{R}_{k}(m, n)=\mathrm{R}_{k-1}(m, n)-\mathrm{C}_{j}(m, n)
$$

Step 5. Order $\mathrm{j}=\mathrm{j}+1$ and repeat Step 2 and Step 3, generating different orders of intrinsic mode components $C_{1}(m, n), C_{2}(m, n), \ldots, C_{j}(m, n)$, until the last remaining component $\mathrm{R}_{\text {res }}(\mathrm{m}, \mathrm{n})$ is the warp direction or weft direction or diagonal direction monotonic variation component. $\mathrm{P}(\mathrm{m}, \mathrm{n})$ can be reconstructed by each intrinsic mode components, expressed as:

$$
\mathrm{P}(m, n)=\sum_{j=1}^{J} \mathrm{C}_{j}(m, n)+\mathrm{R}_{r e s}(m, n)
$$

\section{ALGORITHM DESCRIPTION}

Fabric defect[13] wide range of different types of defects in the empirical mode domains with different local spatial scales. In general, the structure of tissue defects mainly in the first and second order component of the fabric intrinsic mode image. Correspondingly, different defect types of empirical mode[14] domain identification algorithm should have their own characteristics. The main algorithm for four typical types of defects: broken pickss, warfloat, mispicks and hole.

\section{A. Weft Defect Detection}

Weft defect recognition algorithm processes can be described as follows:

Step1. According to 2D-empirical mode decomposition algorithm to decompose the fabric image $\mathrm{P}(\mathrm{m}, \mathrm{n})$, whose size isMXN ,then get it each order intrinsic mode components

$$
\mathrm{C}_{1}(\mathrm{~m}, \mathrm{n}), \mathrm{C}_{2}(\mathrm{~m}, \mathrm{n}), \ldots, \mathrm{C}_{\mathrm{J}}(\mathrm{m}, \mathrm{n})
$$

Step2. Extract the first Order intrinsic mode components $\operatorname{ofC}_{1}(\mathrm{~m}, \mathrm{n})$, then normalize the value of each element to the interval 0 to 1 ,expressed as $\overline{\mathrm{C}}_{1}(m, n) \cdot \operatorname{Sum} \overline{\mathrm{C}}_{1}^{2}(m, n)$ along with warp:

$$
E_{c}(m)=\sum_{n=1}^{N} \overline{\mathrm{C}}_{1}^{2}(m, n)
$$

Step3. Identify the latitude coordinate when $E_{r 2}(n)$ get minimum value, denoted as

$$
n_{\text {opt }}=\arg \min \left[E_{r 2}(n)\right]
$$

Step4. Search all local minimum point of $\overline{\mathrm{C}}_{1}(i,:)$ which is the first $\mathrm{i}$ colum vector of $\overline{\mathrm{C}}_{1}(m, n)$, and then determine the mean spatial scale of $\overline{\mathrm{C}}_{1}(:, j)$, donated as $\Delta_{i c}$.

Step5.Given threshold $\sigma_{\text {th,c }}$ and pixels $p(m, j)$ and $m \in\left[m_{\text {opt }}-\right.$ round $\left.\left(\frac{\Delta_{i c}}{2}\right), m_{o p t}+\operatorname{round}\left(\frac{\Delta_{i c}}{2}\right)\right] \quad$ in which round $(\cdot)$ represents as rounding to the nearest integer. If the expression of $\overline{\mathrm{C}}_{1}(m, j) \leq \sigma_{t h, c}$ is meeted ,labeled this area as pixel defect area.

\section{B. Warfloat Defect Detection}

Recognition by the floating algorithm flow defects can be described as follows:

Step 1. Use 2D-empirical mode decomposition algorithm to decompose the fabric image $\mathrm{P}(m, n)$, whose size is $M \times N$, then get it each order intrinsic mode components $\mathrm{C}_{1}(m, n), \mathrm{C}_{2}(m, n), \ldots, \mathrm{C}_{J}(m, n)$.

Step 2. Extract the first Order intrinsic mode components of $\mathrm{C}_{1}(m, n)$, then normalize the value of each element to the interval 0 to 1 ,expressed as $\overline{\mathrm{C}}_{1}(m, n)$.Sum $\overline{\mathrm{C}}_{1}^{2}(m, n)$ along with zonal:

$$
E_{r 1}(n)=\sum_{m=1}^{M} \overline{\mathrm{C}}_{1}^{2}(m, n)
$$

Step3. Identify the vertical coordinate when $E_{r 1}(n)$ get minimum value, denoted as

$$
n_{o p t}=\underset{n}{\arg \min }\left[E_{r 1}(n)\right] \text {. }
$$

Step4. Search all local minimum point of $\overline{\mathrm{C}}_{1}(i,:)$ which is the first $\mathrm{i}$ zonal vector of $\overline{\mathrm{C}}_{1}(m, n)$ and then determine the mean spatial scale of $\overline{\mathrm{C}}_{1}(:, j)$, donated as $\Delta_{i r}$. 
Step5.Given threshold $\sigma_{t h, r}$ and pixels $p(m, j)$ and $j \in\left[n_{\text {opt }}-\operatorname{round}\left(\frac{\Delta_{i r}}{2}\right), n_{\text {opt }}+\right.$ round $\left.\left(\frac{\Delta_{i r}}{2}\right)\right]$ in which round $(\cdot)$ represents as rounding to the nearest integer. If the expression of $\overline{\mathrm{C}}_{1}(i, j) \leq \sigma_{t h, r}$ is meeted ,labeled this area as pixel defect area.

\section{Hundreds of Feet Defect Identification}

Identify of the hundreds of feet defect algorithm process can be described as follows:

Step 1. 2D empirical mode decomposition algorithm was applied to the size that is $M \times N$ of the fabric image $\mathrm{P}(m, n)$ that extract the first order intrinsic mode components $\mathrm{C}_{1}(m, n)$ and normalize to the range of elements between zero and one. After the normalization of first-order intrinsic mode components are represented as $\overline{\mathrm{C}}_{1}(m, n)$.

Step 2. Assume $\overline{\mathrm{C}}_{1}(m, n)$ that any given the first $\mathrm{j}$ by the vector for $\overline{\mathrm{C}}_{1}(:, j)$, search all the local maximum value and the weft coordinates in $\overline{\mathrm{C}}_{1}(:, j)$.

Step 3. According to $\overline{\mathrm{C}}_{1}(:, j)$ the coordinate calculation of all local maximum values of weft coordinates in the difference sequence of local maximum values $\Delta(k)$, made the value of the smallest element in $\Delta(k)$ is $\Delta_{\min }$.

Step 4. For the first $\tilde{k}$ element $\Delta(\tilde{k})$ in $\Delta(k)$ and preset coefficient $\mu$, to determine whether a conditional expression that is $\Delta(\tilde{k}) \leq \mu \times \Delta_{\min }$ and the other conditional expression that is $\Delta(\tilde{k}+1) \leq \mu \times \Delta_{\min }$ establish at the same time. If $\Delta(\tilde{k}) \leq \mu \times \Delta_{\min }$ and $\Delta(\tilde{k}+1) \leq \mu \times \Delta_{\min }$ is established at the same time, the make coordinate $n=j$ in $\mathrm{P}(m, n)$, all pixels of weft coordinates $\Delta(\tilde{k}-1) \leq m \leq \Delta(\tilde{k}+1)$ are marked as pixels of defect area.

\section{Hole Defect Identification}

The hole defect recognition algorithm can be described as follows:

Step 1. 2D empirical mode decomposition algorithm was applied to the size that is $M \times N$ of the fabric image $\mathrm{P}(m, n)$ that extract the second-order intrinsic mode components $\mathrm{C}_{2}(m, n)$ and normalize to the range of elements between zero and one. After the normalization of second-order intrinsic mode components are represented as $\overline{\mathrm{C}}_{2}(m, n)$.
Step 2. According to the above type for $\overline{\mathrm{C}}_{2}^{2}(m, n)$ to zonal sum:

$$
E_{r 2}(n)=\sum_{m=1}^{M} \overline{\mathrm{C}}_{2}^{2}(m, n)
$$

Step 3. Search the corresponding coordinates when $E_{r 2}(n)$ is the minimum, note for $n_{o p t}=\arg \min \left[E_{r 2}(n)\right]$.

Step 4. Search the minimum value of the $n_{\text {opt }}$ by the elements in $\mathrm{P}(m, n)$, and the minimum value of the weft coordinates is expressed as $m_{o p t}=\underset{m}{\arg \min }\left[\mathrm{P}\left(m, n_{\text {opt }}\right)\right]$.

Step 5. Search scope of weft coordinates within the neighborhood of $m=m_{\text {opt }}$ in $\mathrm{P}(m, n)$, when $m \in \Omega_{m}$, the gray levels of

pixels that are $p\left(m, n_{\text {opt }}\right) \in \mathrm{P}(m, n)$ are less than the given threshold $\tau_{r}$

Step 6. Search by the coordinate range $\Omega_{n}$ within the neighborhood of $n=n_{\text {opt }}$ in $\mathrm{P}(m, n)$, when $n \in \Omega_{n}$, the value of $E_{r 2}(n)$ is less than the given threshold $\tau_{c}$.

Step 7. The given threshold is $\tau$ and pixels are $p(i, j)$, and $(i, j) \in \Omega_{m} \times \Omega_{n}$. If $\overline{\mathrm{C}}_{2}(i, j) \leq \tau$, marked $p(i, j)$ is as pixels of defect area.

\section{Simulation}

In simulation experiment, four kinds of fabric defect images including broken pickss, warfloat, mispicks and hole are showed in Figure II. The results, using 2D empirical mode to decompose this four kinds of fabric defect images ,are showed in Figure III. In Figure III, (a), (b)and (c) are all the first-order intrinsic mode components, and (d) is the second-

order intrinsic mode components. The identity result ofbroken, warfloat, mispicks and hole are showed in figure IV.

The value recognition weft defects when the argument by identifying defects when float is in the range identifying defects latitude and longitude coordinates of the hole, respectively, pre-set various thresholds are shown in Table I.

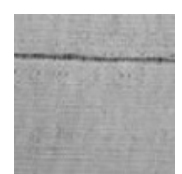

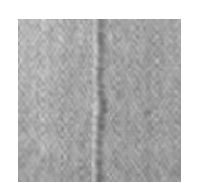

(b) warfloat

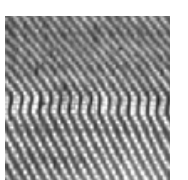

(c) mispickss

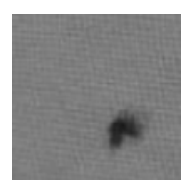

(d) hole FIGURE II. FOUR KINDS OF FABRIC DEFECT IMAGES 


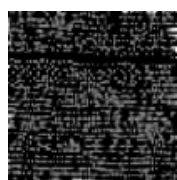

(a) broken picks ( b) warfloat

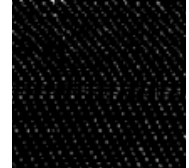

(c) mispickss

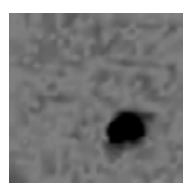

(d) hole
FIGURE III. THE INTRINSIC MODE COMPONENTS OF FABRIC DEFECT IMAGES

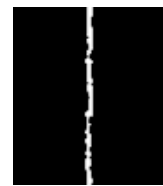

(a) broken picks

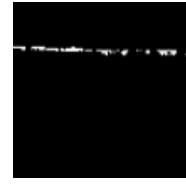

(b) warfloat

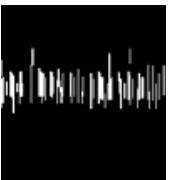

(c) mispicks

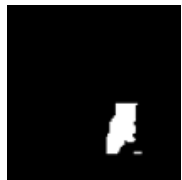

(d) hole
FIGURE IV. FOUR KINDS OF IDENTITY RESULTS OF FABRIC DEFECTS

TABLE I. DECISION THRESHOLD OF FOUR KINDS OF FABRIC DEFECT

\begin{tabular}{|c|c|c|c|c|}
\hline $\begin{array}{c}\text { defect } \\
\text { type }\end{array}$ & $\sigma_{t h, c}$ & $\sigma_{t h, r}$ & $\mu \times \Delta_{\mathrm{mi}}$ & $\tau$ \\
\hline $\begin{array}{c}\text { Broken } \\
\text { picks }\end{array}$ & 0.333 & - & - & - \\
\hline warfloat & - & 0.333 & - & - \\
\hline mispicks & - & - & 6 & - \\
\hline hole & - & - & - & 0.33 \\
\hline
\end{tabular}

\section{CONCLUSION}

The essential characteristics of $2 \mathrm{D}$ empirical mode decomposition method is based on the image inherent spatialthe image to be processed is divided into different orders of intrinsic mode components. Thus, for fabric defect of a warp or weft fine scale, its organizational structure information is mainly contained in the first order of intrinsic mode components. As for the main energy distribution in the band of fabric defect, its organizational structure information is mainly contained in the second order of intrinsic mode components. Based on the weft, floating by, a hundred feet and the hole in the fabric sample 2D defects empirical mode decomposition to the empirical mode decomposition after the first or second intrinsic mode components warp or weft fabric defect characterization of energy local feature structure, not only can adaptively change the fabric background tissue, and can portray fine fabric defects inherent spatial scale change. Simulation results show that this method can effectively identify the weft, floating by, a hundred feet and holes and other defects typical fabrics, the algorithm is simple and fast operation, to build a line of fabric defects identification system provides an important technical reference.

\section{ACKNOWLEDGMENT}

This work is supported by the Graduate Student Innovation Project of in 2016 (No. 120301990122) and the Education Teaching Reform Project in 2015 (No.ZDJG-1506) of Beijing Institute of Fashion Technology.

\section{REFERENCES}

[1] Srinivasan K, Dastor P H, Radhakrishnaihan $\mathrm{P}$ et al. FDAS:A knowledge-based frame detection work for analysis of defects in woven textile structures . J. Text. Inst., 1992, 83
[2] Zhang Y F, Bresee R R. Fabric defect detection and classification using image analysis. Textile Research Journal, 1995, 65(1)

[3] Chetverikov Dmitry. Pattern regularity as a visual key. Image and Vision Computing, 2000, 18(12)

[4] Rösler R N U. Defect detection in fabrics by image processing. Melliand-Text.ber., 1992, 73

[5] Tsai I, Lin C, Lin J. Applying an artificial neural network to pattern recognition in fabric defects. Textile Research Journal, 1995, 65(3)

[6] Randen T. Filtering for texture classification: a comparative study. IEEE Transactions on Pattern Analysis and Machine Intelligence, 1999, 21(4)

[7] Chan C H, Pang G. Fabric defect detection by Fourier analysis. IEEE Transactions on Industry Applications, 2000, 36(5)

[8] Campbell J G, Murtagh F. Automatic visual inspection of woven textiles using a two-stage defect detector. Optical Engineering, 1998, 37(9)

[9] Jasper W, Garnier S, Potlapalli H. Texture characterization and defect detection using adaptive wavelets. Optical Engineering, 1996, 35(11)

[10] Kumar A, Pang G. Identification of surface defects in textured materials using wavelet packets. Thirty-Sixth IAS Annual Meeting, 2001(1)

[11] Kuma A, Pang G. Fabric defect segmentation using multichannel blob detectors. Optical Engineering, 2000, 39 (12)

[12] Nunes J C, Bouaoune Y, Delechelle E. Image analysis by bidimensional empirical mode decomposition. Image and Vision Computing, 2003(21)

[13] Nunes J C, Guyot S, Delechelle E. Texture analysis based on local analysis of the Bidimensional Empirical Mode Decomposition. Machine Vision and Applications, 2005, 16

[14] Xu Y, Liu B, Liu J, Riemenschneider S. Two-dimensional empirical mode decomposition by finite elements. Proceedings of the Royal Society London A, 2006, 462 